\title{
OTIMIZAÇÃO DE PROCESSO PRODUTIVO ATRAVÉS DA MINERAÇÃO DE DADOS
}

LIMA, Anderson Luiz Ignacio de

Instituto Federal Fluminense (IFF)

R. Dr. Siqueira, 273 - Parque Dom Bosco, Campos dos Goytacazes - RJ, 28030-130

igdelima@gmail.com

PEREIRA, Bruno Leonardo Silva

Instituto Federal Fluminense (IFF)

R. Dr. Siqueira, 273 - Parque Dom Bosco, Campos dos Goytacazes - RJ, 28030-130

bruno4cantos@yahoo.com.br

HORA, Henrique Rego Monteiro da

Instituto Federal Fluminense (IFF)

R. Dr. Siqueira, 273 - Parque Dom Bosco, Campos dos Goytacazes - RJ, 28030-130

henrique.dahora@iff.edu.br

MORAIS, Alline Sardinha Cordeiro

Instituto Federal Fluminense (IFF)

R. Dr. Siqueira, 273 - Parque Dom Bosco, Campos dos Goytacazes - RJ, 28030-130

allinescmorais@yahoo.com.br

\section{RESUMO}

Atualmente em todos os setores a concorrência se destaca como um desafio que tem que ser superado a cada instante pelas corporações. Nesse sentido qualquer que sejam os diferenciais competitivos desenvolvidos, podem trazer grandes ganhos relacionados à produtividade. Este estudo mostra como uma inovação aplicada através da ciência de Mineração de Dados pode trazer enormes ganhos num processo produtivo, otimizando custos, diminuindo o tempo de resposta e aumentando a assertividade nas tomadas decisão.

Palavra-chave: Mineração de Dados; Otimização; Produtividade; Processos.

\begin{abstract}
Currently in all sectors the competition stands out as a challenge that has to be overcome at every moment by the corporations. In this sense whatever the competitive differentials developed, can bring great gains related to productivity. This study shows how an innovation applied through the science of Data Mining can bring enormous gains in a productive process, optimizing costs, reducing response time and increasing assertiveness in decision making.
\end{abstract}

Keywords: Data mining; Optimization; Productivity; Process. 


\section{Como Citar:}

LIMA, Anderson Luiz Ignacio de et al. Otimização de processo produtivo através de Mineração de Dados. In: SIMPÓSIO DE PESQUISA OPERACIONAL E LOGÍSTICA DA MARINHA, 19., 2019, Rio de Janeiro, RJ. Anais [...]. Rio de Janeiro: Centro de Análises de Sistemas Navais, 2019.

\section{INTRODUÇÃO}

De acordo com Juran \& Godfrey [8] existem muitos significados para a palavra qualidade e dentre estes, dois são tidos como críticos para a gestão da qualidade. O primeiro é que significa as características dos produtos que atendem às necessidades do cliente e, portanto, satisfação do cliente. No entanto, fornecer mais ou fornecer melhores recursos de qualidade geralmente requer um investimento e isso geralmente envolve aumentos nos custos. O segundo significado para a qualidade é a ausência de deficiências, ou seja, livres de erros que requerem algum tipo de retrabalho. O gerenciamento contínuo de falhas que levem a algum tipo de retrabalho que impacta diretamente na insatisfação do cliente.

Para Porter [12] a essência de uma estratégia competitiva é relacionar uma companhia ao seu meio ambiente. Mesmo este meio ambiente relevante sendo muito amplo, abrangendo forças econômicas e sociais, o aspecto principal para a empresa é a indústria os as indústrias em que ela compete. Nesse sentido o desenvolvimento de uma estratégia competitiva é a criação de uma fórmula ampla para o modo como uma empresa irá competir e quais deveriam ser as metas e políticas necessárias para levá-la a seus objetivos.

A fim de alcançar estas metas, empresas de ponta, que buscam se diferenciar pela sua tecnologia tem sempre que estar pensando em qual será o próximo passo, ou seja, como irão inovar para continuarem se diferenciando de seus concorrentes.

Neste contexto, a inovação vem muitas vezes com objetivos estratégicos, como a melhoria da imagem da organização, renovação do portfólio de produtos, aumento da produtividade, upgrades de processo, etc. De acordo com Spezamiglio et al. [15] existe uma relação positiva entre inovação e competitividade, garantindo às empresas uma posição de mercado rentável e favorável através de uma estratégia difícil de ser imitada e criando uma barreira a novos entrantes.

Neste artigo o objetivo é inovar um processo produtivo utilizando como ferramenta a Mineração de Dados, criando um diferencial competitivo num processo de produção. Gerando um conjunto de dados e informações que serão utilizados para antever uma falha no processo, evitando uma perda de qualidade, no custo de produção, no volume de produção e na oportunidade de se otimizar o plano de produção.

\section{BASE CONCEITUAL}

De acordo com Amaral [1] os dados são fatos coletados e armazenados, a informação é o dado analisado e com algum significado e o conhecimento é a informação interpretada, entendida a aplicada para um fim. Ressalta também que o fenômeno Big Data, onde os dados são produzidos em vários formatos e armazenados por uma grande quantidade de dispositivos e equipamentos, será uma vantagem competitiva no core business das empresas e criação de produtos ou serviços orientados a dados.

O Processo de Extração de Conhecimento, também conhecido pelo termo em inglês Knowledge Discovery in Databases (KDD), tem como uma etapa importante a Mineração de Dados. Segundo Berson, Smith \& Thearling [3], a Mineração de Dados explora as bases de dados através de dezenas de centenas de pontos de vista diferentes. A diferença entre a Mineração de Dados e as Técnicas Estatísticas está na utilização dos próprios dados para a descoberta dos padrões e não na verificação de padrões hipotéticos. 
As bases de dados armazenam conhecimento que podem auxiliar na melhoria dos negócios, e as técnicas tradicionais permitem somente verificar hipóteses que são, aproximadamente, apenas $5 \%$ de todas as relações encontradas por esses métodos. A Mineração de Dados pode descobrir as outras relações desconhecidas: os 95\% restantes [2].

\subsection{EXTRUSÃO}

A técnica da extrusão é a mais utilizada atualmente e permite a fabricação de peças plásticas de qualidade, através de um processo contínuo e de alta produtividade. Alguns dos produtos fabricados a partir desse método são: tubos, perfis, mangueiras, revestimentos de fios e cabos, chapas, filmes/bobinas, embalagens, sacos e sacolas.

Diferentemente da injeção, o processo de extrusão é utilizado para dar forma final aos produtos, que saem da extrusora semi-acabados. Considerado o mais importante processo de transformação de plásticos, a extrusão consiste em solidificar o termoplástico previamente aquecido, por meio da água. Nesse processo, a passagem do material granulado é forçada por meio de um cilindro aquecido, através de uma ou duas roscas que giram e enviam material o tempo todo para a matriz.

Extrudar é forçar a passagem de um material através de um orifício. Na indústria em geral, a extrusão de um material é usada para dar forma a ele e conferir determinadas características.

Nas indústrias metalúrgicas ou automobilísticas, a extrusão é um processo de conformação mecânica (ou conformação plástica) de materiais plásticos ou metálicos, assim como a trefilação, laminação ou estampagem (terminologias usadas para metais). Podem ser extrudadas peças longas com a seção transversal no formato que se desejar e, posteriormente, secioná-las de modo a produzir diversas peças com a mesma seção transversal de uma só vez.

Nesse processo a peça é conformada pela ação combinada de tensões (tração e compressão), mas o que faz com que a peça adquira o formato desejado é a resistência imposta pela matriz (molde ou orifício) à passagem da peça. Assim, é dito que a extrusão é um processo de conformação onde a força predominante é a compressão indireta (desenvolvida pela matriz).

Os polímeros termoplásticos (aqueles que podem ser amolecidos com o aumento da temperatura) também podem ser extrudados pelo método hidrostático fluido a fluido dando origem a tubos, bastões e diversos tipos de materiais. Os tipos de plásticos utilizados no processo de extrusão são: polietileno de baixa ou alta densidade (PEBD e PEAD), o vinil, o PVC, acrílico, PETG, butirato, polipropileno e poliestireno. O processo de extrusão pode ser melhor entendido através da figura 2.1.

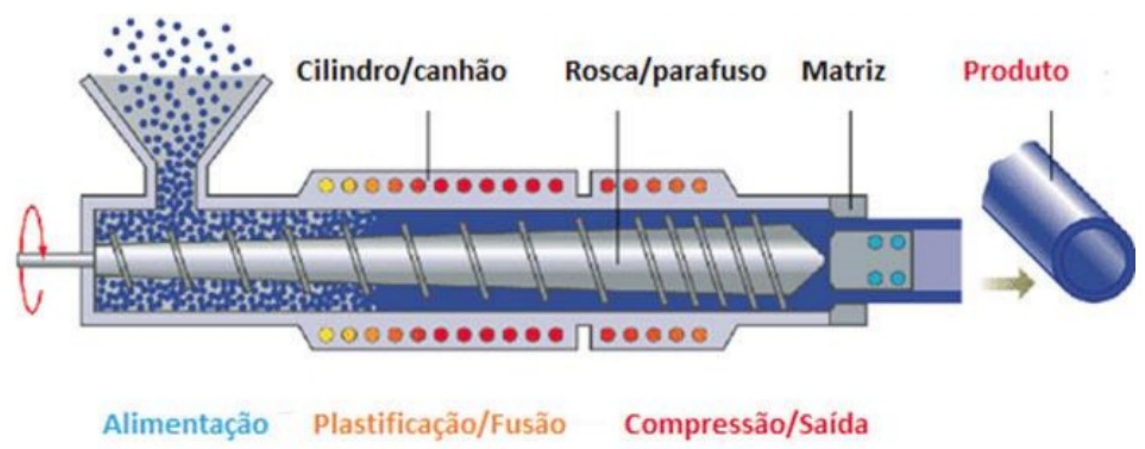

Figura 2.1 - Processo de extrusão [17]. 
Controles dimensionais são executados de modo a garantir a padronização e uniformidade da produção, e para isso um equipamento de grande auxílio é o Equipamento de Medição ultrassônica online de espessura (Figura 2.2), onde o equipamento faz a leitura dimensional do produto a través de ondas ultrassônicas e apresenta esses através de IHM para acompanhamento da produção, além de armazenar esses dados para posterior consulta.

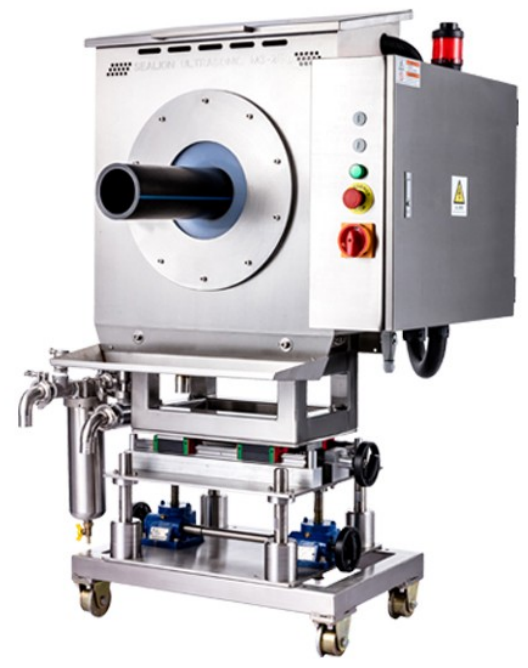

Figura 2.2 - Equipamento de Medição ultrassônica online de espessura [16].

\section{OBJETIVO}

Este trabalho tem como objetivo inovar um processo de produção através da utilização de técnicas de Mineração de Dados para otimização de resultados produtivos através da identificação de tendências de variações dimensionais em linhas de extrusão para que ações de correção sejam tomadas antes dos valores saírem da especificação. O que pode levar a ganhos financeiros provenientes de uma eliminação do risco de se interromper uma produção decorrente de um produto fora da especificação.

\section{METODOLOGIA}

Esta seção, descreve as etapas utilizadas para aquisição dos dados, explica o préprocessamento e a aplicação das ferramentas de Mineração de Dados, detalhando a aplicação do método de classificação e técnicas de análises utilizadas.

\subsection{AQUISIÇÃo DE DADOS}

Os dados utilizados para realização do presente trabalho foram fornecidos pela empresa onde os dados foram gerados e que, por opção da mesma, terá seu nome omitido.

Os dados são gerados a partir de medições online através de equipamento ultrassônico de medição e apresentados em tempo real para o usuário da máquina através de um IHM (Interface Homem-Máquina). O equipamento faz medições a cada 1 segundo de produção, e esses dados são ainda armazenados e podem ser extraídos para análise futura.

Foram coletados dados históricos de produções anteriores para em seguida aplicar as técnicas de Mineração de Dados e assim avaliar a viabilidade de sua utilização. 


\subsection{Pré-Processamento dos Dados}

A etapa do pré-processamento dos dados, segundo Han \& Kamber [6] pertence ao Processo de Extração de Conhecimento - KDD, onde a mineração de dados é uma etapa importante. Após a seleção dos dados e antes da aplicação das técnicas de mineração de dados, existe a etapa de seleção e transformação. Nesta etapa os atributos originais da base de dados são convertidos e/ou adaptados para aplicar a mineração de dados.

$\mathrm{O}$ arquivo gerado pelo equipamento de medição online gera um arquivo Excel com todas as medições sem indicar o resultado dessa medição, ou seja, não correlaciona a medição feita com a especificação do produto. Deste modo, foi incluído um campo de "OK/ NOK" para rotular o desfecho da medição entre os possíveis resultados de dentro ou fora da especificação. Tal rotulagem foi definida em função das especificações do produto onde determina "OK" como resultados de medições onde os valores encontrados estão dentro das limítes de especificação, e "NOK" para os resultados de medições onde os valores encontrados estão fora dos limites de especificação do produto. As especificações do produto são definidas por documento disponível pela Engenharia de Produto para a o Setor de Produção. Após esse tratamento, foi gerado um arquivo .csv para leitura pelo software de análise.

As medições são feitas em 8 pontos equidistantes ao longo da circunferência do produto, conforme mostrado na figura abaixo.

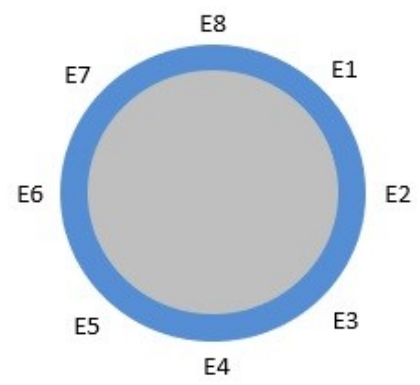

Figura 4.3 - Representação das posições de medições das espessuras.

\subsection{Procedimentos Metodológicos e téCNICAS de ANÁlises}

Os algoritmos foram executados a partir da ferramenta de mineração de dados WEKA, detalhes em Hall et al. [7] e Bouckaert [4], ela possui algoritmos de aprendizado de máquina que podem ser utilizados para extrair informações relevantes de uma base de dados. A ferramenta foi adotada, de acordo com os seguintes motivos: i) ferramenta open-source e livre de custos; ii) possuir várias versões de algoritmos empregados na mineração de dados; iii) disponibilidade de recursos estatísticos para comparar o desempenho dos algoritmos e apresentar diversos recursos para análise dos dados.

O algoritmo classificador selecionado foi o J48, que é uma implementação de código aberto em Java do algoritmo C4.5 [13] no programa Weka [7], que gera árvores de classificação a partir de um conjunto de dados de treinamento, sendo que, a cada nó, o algoritmo escolhe um atributo que mais eficientemente subdivide o conjunto das amostras em subconjuntos homogêneos e caracterizados por sua classe. O critério é o ganho de informação obtida na escolha do atributo para subdivisão [13; 7]. O algoritmo J48 foi considero um dos mais utilizados em mineração de dados segundo $\mathrm{Wu}$ et al. [18] e descrito em Top 10 algorithms in data mining. 


\section{RESULTADOS}

Nesta seção, são apresentados os resultados obtidos após a aplicação do algoritmo classificador J48 sobre a base de dados estudada.

Abaixo é apresentado a vista do software WEKA com o resultado do classificador J48.

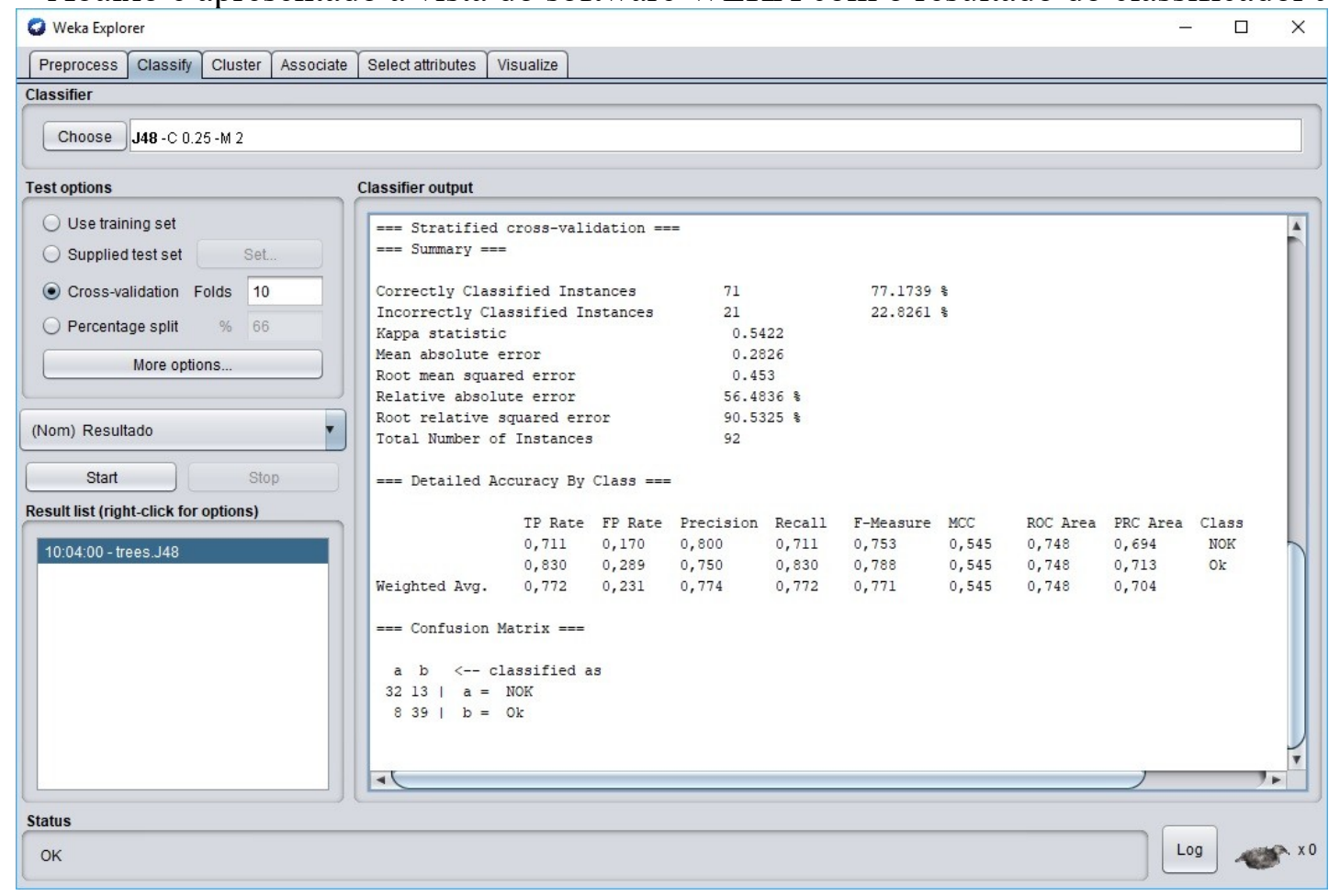

Figura 5.4 - Vista do software Weka.

As acurácias obtidas para os dados utilizados foram de 80,0 \% para classificação NOK e de $75,0 \%$ para a classificação $\mathrm{OK}$, isso quer dizer que em média o nível de acerto na classificação foi de $77,4 \%$, conforme pode ser visto na tabela abaixo.

\begin{tabular}{|c|c|c|c|c|c|c|c|c|c|}
\hline & TP Rate & FP Rate & Precision & Recall & F-Measure & MCC & ROC Area & PRC Area & Class \\
\hline & 0,711 & 0,170 & 0,800 & 0,711 & 0,753 & 0,545 & 0,748 & 0,694 & NOK \\
\hline & 0,830 & 0,289 & 0,750 & 0,830 & 0,788 & 0,545 & 0,748 & 0,713 & $\mathrm{OK}$ \\
\hline Weighted Avg. & 0,772 & 0,231 & 0,774 & 0,772 & 0,771 & 0,545 & 0,748 & 0,704 & \\
\hline
\end{tabular}

Tabela 1 - Detalhamento das acurácias.

Na matriz de confusão gerada (Tabela 2) pode se observar os acertos e erros encontrados na classificação, conforme mostrado abaixo. Desde modo, 32 observações foram classificadas como "NOK" e realmente deveriam ser classificadas como "NOK" (negativo verdadeiro); 13 observações foram classificadas como "OK", porém são "NOK" (falso positivo); 8 observações foram classificadas como "NOK, porém são "OK" (falso negativo) e 39 observações foram classificadas como "OK" e realmente deveriam ser classificadas como tal (positivo verdadeiro). 


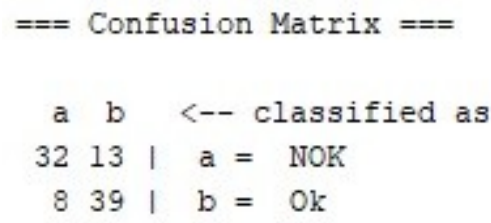

Tabela 2 - Matriz de confusão.

Através da Árvore de Decisão é possível inferir que dentre os 8 pontos de medição, E1 é uma posição crítica e que caso seu valor fique abaixo de $\sim 11,20 \mathrm{~mm}$ poderá causar problemas, tornando-o um ponto de atenção.

Caso o valor de E1 se mantenha acima de $\sim 11,20 \mathrm{~mm}$ então o próximo ponto em que se deve tomar cuidado é o ponto E5, em que caso o valor fique abaixo de $\sim 10,06 \mathrm{~mm}$ então passa a ser problema, e caso fique acima precisa-se ficar atento ao ponto E7.

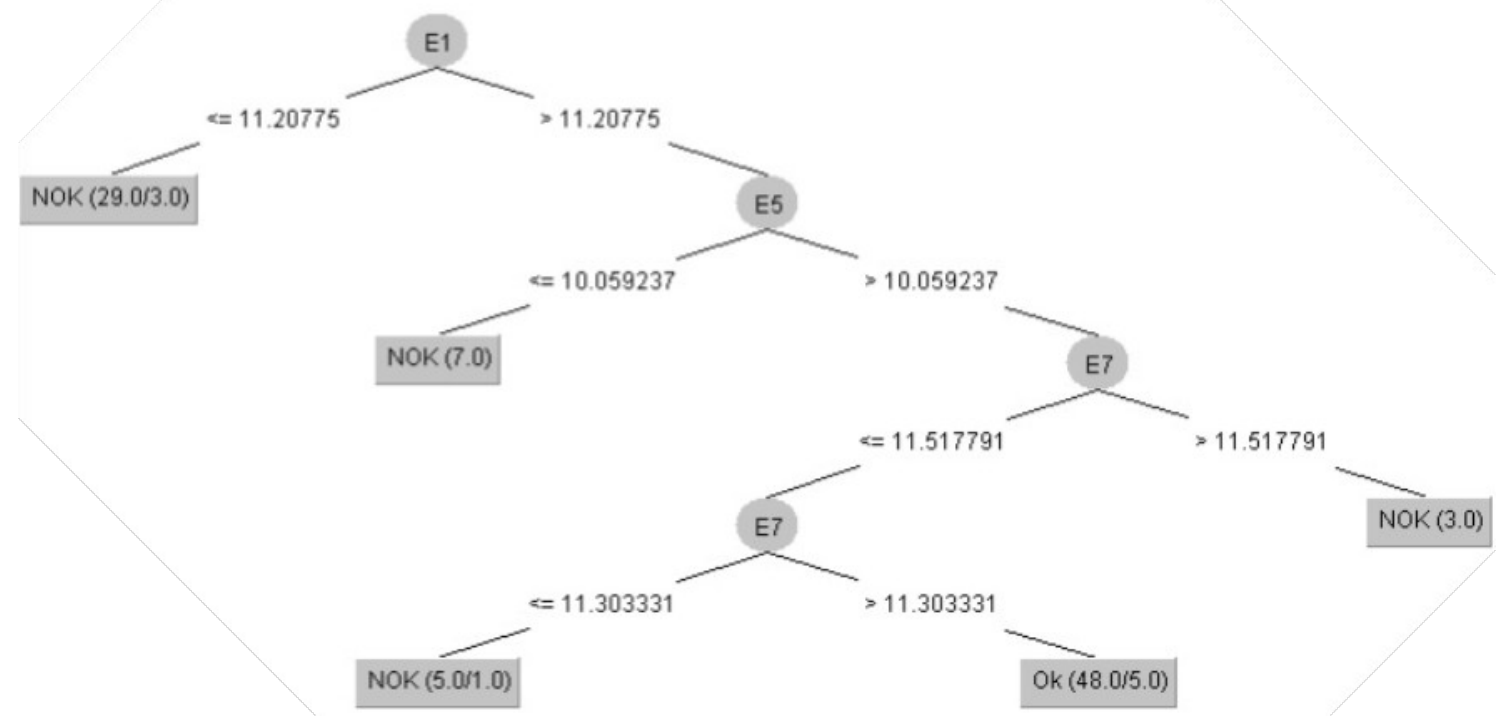

Figura 5.5 - Árvore de decisão.

\section{CONCLUSÃO}

A potencial mudança dos negócios de uma empresa destacam novos direcionamentos que são frequentemente necessários para vincular o desempenho econômico e o progresso nas principais atividades da organização. Nesse sentido a necessidade inovação seja ela em produto, serviço, processo, marketing, organizacional etc., fomenta um diferencial competitivo gerando valor para a organização.

De acordo com os resultados obtidos, podemos concluir que é possível encontrar uma tendência de resultado em função dos dados gerados através das medições online.

Esse estudo pode ser integrado à produção e com isso minimizar os riscos de desvios durante a produção, além de se obter maior estabilidade do processo. Além da redução de custos de matéria prima advindo de um melhor controle e a percepção de maior qualidade do produto. 


\section{REFERÊNCIAS BIBLIOGRÁFICAS}

[1] AMARAL, F. Introdução à Ciência de Dados: Mineração de Dados e Big Data. Rio de Janeiro: Alta Books, 2016.

[2] BARBOSA, D. C. C.; MACHADO, M. A. Mineração de Dados usando o software WizRule em Base de Dados de Compras de TI. Revista Eletrônica de Sistemas de Informação, 6(1), 2007.

[3] BERSON, A.; SMITH, S.; THEARLING, K. Building data mining applications for CRM. New York, NY: McGraw-Hill, 2000.

[4] BOUCKAERT, R.; EIBE F.; MARK H. (et al.). WEKA Manual for Version 3-6-4. December 2010.

[5] FERNANDO, A. Introdução à Ciencia de Dados: mineração de dados e big data. [s.1.] Alta Books Editora, 2016.

[6] HAN, J.; KAMBER, M. Data Mining Concepts and Techniques. 2nd. ed. Morgan Kauffmann Publishers, Second Edition, 2006

[7] HALL, M.; FRANK, E., HOLMES, G. (et al.). The WEKA Data Mining Software: An Update. SIGKDD Explorations, v. 11, n. 1, 2009.

[8] JURAN, J. M.; GODFREY, A. B. (eds.). Juran's quality handbook. 5th. ed. New York: McGraw Hill, 1999.

[9] LICHTENTHALER, U. Shared Value Innovation: Linking Competitiveness and Societal Goals in the Context of Digital Transformation. International Journal of Innovation and Technology Management, v. 14, n. 4, p. 1750018, ago. 2017. Disponível em: https://www.worldscientific.com/doi/pdf/10.1142/S0219877017500183. Acesso em: 02 dez. 2018.

[10] MARQUES, A. B. et al. A relação entre competências para inovar e competitividade na indústria de eletromédicos no Brasil. Gestão \& Produção, v. 25, n. 3, p. 545-556, 23 abr. 2018.

[11] PETRAKIS, P. E.; KOSTIS, P. C.; VALSAMIS, D. G. Innovation and competitiveness: Culture as a long-term strategic instrument during the European Great Recession. Journal of Business Research, v. 68, n. 7, p. 1436-1438, jul. 2015.

[12] PORTER, M. E. (Org.). Competition in global industries (Nachdr.). Boston, Mass: Harvard Business School Press, 1993.

[13] QUINLAN, J. R.; C4.5 programs for machine learning. San Francisco: Morgan Kaufmann Publishers, 1993.

[14] RIEG, D. L.; ALVES FILHO, A. G. Esforço tecnológico e desempenho inovador das 
empresas do setor médico-hospitalar localizadas em São Carlos, SP. Gestão \& Produção, v. 10, n. 3, p. 293-310, dez. 2003. Disponível em: http://www.scielo.br/pdf/gp/v10n3/19164.pdf. Acesso em: 3 dez. 2018.

[15] SPEZAMIGLiO, B. DOS S.; GAlinA, S. V. R.; CALIA, R. C. Competitividade, inovação e sustentabilidade: uma inter- relação por meio da sistematização da literatura. REAd, Revista Eletrônica de Administração (Porto Alegre), v. 22, n. 2, p. 363-393, ago. 2016. Disponível em: http://www.scielo.br/pdf/read/v22n2/1413-2311-read-22-0200363.pdf. Acesso em: 2 dez. 2018.

[16] SEALION. SL-meas315|ultrasonic on-line thickness measuring system. Disponível em: $\quad$ http://www.sealion-china.com/on-Line-ultrasonicuthickness-measuring/250250.html. Acesso em: $11 \mathrm{dez} .2018$.

[17] STÁBIL PARTNER TECH. Qual a diferença de injeção e extrusão? Disponível em: http://stabil.ind.br/qual-a-diferenca-de-injecao-e-extrusao. Acesso em: 11 dez. 2018.

[18] WU, X.; KUMAR, V.; ROSS, Q. J.(et al.) Top 10 algorithms in data mining. Journal of Knowledge and Information Systems, v. 14, n. 1, p. 1-37, 2008. 